\title{
CYLD-mutant cylindroma-like basaloid carcinoma of the anus: a genetically and morphologically distinct class of HPV-related anal carcinoma
}

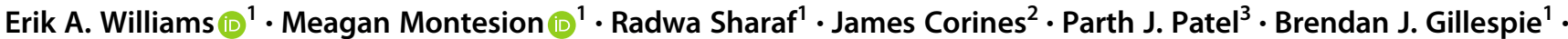 \\ Dean C. Pavlick ${ }^{1} \cdot$ Ethan S. Sokol $^{1} \cdot$ Brian M. Alexander $^{1} \cdot$ Kevin Jon Williams $^{4} \cdot$ Julia A. Elvin $^{1} \cdot$ Jeffrey S. Ross ${ }^{1,2}$.

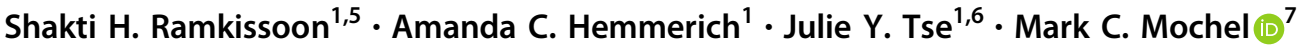

Received: 19 April 2020 / Revised: 17 May 2020 / Accepted: 18 May 2020 / Published online: 27 May 2020

(c) The Author(s) 2020. This article is published with open access

\begin{abstract}
Rare reports of anal carcinoma (AC) describe histologic resemblance to cutaneous cylindroma, but mutations in the tumor suppressor $C Y L D$, the gene responsible for familial and sporadic cylindromas, have not been systematically investigated in AC. Here, we investigate $C Y L D$-mutant $\mathrm{AC}$, focusing on molecular correlates of distinct histopathology. Comprehensive genomic profiling (hybrid-capture-based DNA sequencing) was performed on 574 ACs, of which 75 unique cases (13\%) harbored a $C Y L D$ mutation. Clinical data, pathology reports, and histopathology were reviewed for each $C Y L D$-mutant case. The spectrum of $C Y L D$ mutations included truncating $(n=50 ; 67 \%)$, homozygous deletion $(n=10 ; 13 \%)$, missense $(n=16 ; 21 \%)$, and splice-site $(n=3 ; 4 \%)$ events. Compared with CYLD-wildtype AC $(n=499), C Y L D$-mutant ACs were significantly enriched for females ( $88 \%$ vs. $67 \%, p=0.0001$ ), slightly younger (median age 59 vs. 61 years, $p=0.047$ ), and included near-universal detection of high-risk HPV sequences (97\% vs. 88\%, $p=0.014)$, predominantly HPV16 $(96 \%)$. The $C Y L D$-mutant cohort also showed significantly lower tumor mutational burden (TMB; median 2.6 vs. $5.2 \mathrm{mut} / \mathrm{Mb}, p<0.00001$ ) and less frequent alterations in PIK3CA (13\% vs. $31 \%, p=0.0015)$. On histopathologic examination, $73 \%$ of $C Y L D$-mutant AC (55/75 cases) showed a striking cylindroma-like histomorphology, composed of aggregates of basaloid cells surrounded by thickened basement membranes and containing characteristic hyaline globules, while only $8 \%$ of $C Y L D$-wildtype tumors $(n=34 / 409)$ contained cylindroma-like hyaline globules $(p<0.0001)$. CYLD-mutant carcinomas with cylindroma-like histomorphology $(n=55)$ showed significantly lower TMB compared with $C Y L D$-mutant cases showing basaloid histology without the distinctive hyaline globules $(n=14)$ (median 1.7 vs. $4.4 \mathrm{mut} / \mathrm{Mb}, p=0.0058)$. Only five $C Y L D$-mutant cases (7\%) showed nonbasaloid conventional squamous cell carcinoma histology (median TMB $=5.2 \mathrm{mut} / \mathrm{Mb}$ ), and a single $C Y L D$-mutant case showed transitional cell carcinoma-like histology. Within our cohort of ACs, CYLD mutations characterize a surprisingly large subset (13\%), with distinct clinical and genomic features and, predominantly, a striking cylindroma-like histopathology, representing a genotype-phenotype correlation which may assist in classification of AC.
\end{abstract}

\section{Introduction}

Most cancers of the anal and perianal region are squamous cell carcinomas (SCC), predominantly HPV-related [1-4]. Previous publications have reported rare occurrences of anal

Supplementary information The online version of this article (https:// doi.org/10.1038/s41379-020-0584-2) contains supplementary material, which is available to authorized users.

Erik A. Williams

erwilliams@foundationmedicine.com

Extended author information available on the last page of the article carcinomas (AC) with "adenoid cystic carcinoma-like" or "cylindroma-like" histologic features, including dense hyaline inclusions of basement membrane material within tumor lobules resembling cutaneous cylindroma [5-7]. The current WHO classification of digestive system tumors acknowledges basaloid patterns in AC, including rare adenoid cystic carcinoma-like histologic features, but describes "the abandonment of histologic subtyping for clinical purposes" owing to intratumoral heterogeneity, limited sampling in small biopsies, and subjectivity [1].

Here, we hypothesized that a molecular approach would allow us to identify these cases and improve the classification system. To address our hypothesis, we chose to focus 
on mutations in the tumor suppressor $C Y L D$, the gene responsible for both familial [8] and sporadic [9] cylindromas. Recent molecular genetic analysis of HPV-positive head and neck SCC has shown the significance of CYLD mutation in HPV-driven neoplasia [10]. CYLD normally functions as a deubiquitinating enzyme that negatively regulates the $\mathrm{NF}-\kappa \mathrm{B}[11,12]$ and $\mathrm{c}$-Jun $\mathrm{N}$-terminal kinase pathways [13-15]. A study of HPV-positive head and neck SCCs found that CYLD-mutant cases showed increased NF$\kappa \mathrm{B}$ activity, episomal HPV DNA instead of integrated HPV DNA, and improved survival, although histopathologic data were not reported [10].

In the current study, we performed a retrospective analysis of AC samples analyzed by comprehensive genomic profiling (CGP) for tumors with CYLD mutations. Surprisingly, $13 \%$ of our cohort of ACs carried CYLD mutations, and those tumors show near-universal detection of high-risk HPV sequences, low tumor mutational burden, and, predominantly, a striking cylindroma-like histopathology with characteristic hyaline globule inclusions.

\section{Materials and methods}

\section{Cohort and genomic analyses}

The Western Institutional Review Board (Protocol No. 20152817) approved this study, including an informed consent waiver and a HIPAA waiver of authorization. A database of cases, which had undergone CGP as part of routine clinical care, performed in a Clinical Laboratory Improvement Amendments-certified, College of American Pathologistsaccredited laboratory (Foundation Medicine, Inc., Cambridge, MA, USA), was queried for cases of AC with CYLD mutation. The pathologic diagnosis of each case was confirmed on routine hematoxylin and eosin $(\mathrm{H} \& \mathrm{E})$-stained slides before DNA extraction. Sections were macrodissected to achieve an estimated percent tumor nuclei (\%TN) above $20 \%$ in each case, where $\% \mathrm{TN}=100$ times the number of tumor cells divided by total number of nucleated cells. For genomic analysis, $\geq 60 \mathrm{ng}$ DNA was extracted from $40-\mu \mathrm{m}$ sections of tumor samples in formalin-fixed, paraffin-embedded tissue blocks. The samples were assayed by adaptor ligation hybrid capture, performed using the Foundation One T7 baitset [16]. All sequenced genes are listed in Supplemental Table 1. Sequencing of captured libraries was performed using the Illumina HiSeq 4000 System to a mean exon coverage depth of targeted regions of $>500 \times$, and sequences were analyzed for genomic alterations, including short variant alterations (base substitutions, insertions, and deletions), copy number alterations (focal amplifications and homozygous deletions), and select gene fusions or rearrangements [16-18]. To maximize mutation detection accuracy (sensitivity and specificity) in impure clinical specimens, sequencing was previously optimized and validated to detect base substitutions at a $\geq 5 \%$ mutant allele frequency (MAF), indels with a $\geq 10 \% \mathrm{MAF}$ with $\geq 99 \%$ accuracy, and fusions occurring within baited introns/exons with $>99 \%$ sensitivity [16]. Tumor mutational burden (TMB, mutations/Mb) was calculated based on sequences of 0.8-1.1 Mbp of DNA [18]. Up to 114 loci were assessed for microsatellite instability determinations [19]. For detection of HPV genome sequences, BLASTn comparison of de novo assembly of nonhuman sequencing reads was performed against all viral nucleotide sequences in the comprehensive NCBI RefSeq database. Various HPV types were assessed, including HPV6, 11, 16, 18, 26, 31, 33, 35, 39, 40, $42,43,44,45,51,52,53,54,55,56,58,59,61,62,64,66$, 67, 68, 69, 70, 71, 72, 73, 81, 82, 83, 84, CP6108, and IS39. HPV types were classified as described by Muñoz et al. [20], with HPV16, 18, 33, and 58 labeled high risk. $\operatorname{HPV}(+)$ designation required contigs $\geq 80$ nucleotides in length with $\geq 97 \%$ sequence identity to the BLAST database sequence.

\section{Mutational signatures}

Characterization of mutational signatures was performed for all specimens with at least 20 nondriver somatic missense alterations. Mutational signatures were designated by trinucleotide context analysis using the Sanger COSMIC cancer mutational signatures [21]. A positive signature required a sample to have at least a $40 \%$ fit to a known mutational process, including APOBEC overexpression, exposure to ultraviolet light, hypofunction of the BRCA tumor suppressor, and defects in mismatch repair [21].

\section{Germline prediction}

Identification of $C Y L D$ mutations as likely to be germline, rather than somatic, was performed using a validated somaticgermline-zygosity algorithm as previously described [17]. In brief, the alignment of sequencing reads and mutant allele frequencies for mutations detected by the Foundation Medicine sequencing pipeline was compared with the expected values produced by the copy number model [16]. Based on this comparison, the algorithm generated a prediction of whether the variant was germline, somatic, or ambiguous [17]. This computational method was utilized instead of the optimal approach of comparative sequencing of paired normal tissue, which was unavailable.

\section{Clinical-pathological analysis of anal carcinoma cohort harboring CYLD mutation}

Tumor samples for CGP (Foundation Medicine, Cambridge, MA, USA) had been collected from patients receiving clinical care at other institutions. Clinical- 
pathological data, including patient age, gender, tumor site, and AJCC stage (8th edition) [22] were collected from accompanying pathology reports.

H\&E stained sections from each $C Y L D$-mutant case were assessed retrospectively by three pathologists (EAW, JYT, MCM), with the exception of a single case where slides were not available. Histologic features were evaluated, including growth pattern (diffuse, nodular, corded, etc.), glandular differentiation, peripheral palisading of nuclei, presence and arrangement of basement-membrane material, necrosis, presence of squamous differentiation (squamous pearls or eddies), and cytomorphology (basaloid, squamoid, etc.). Accompanying pathology reports were utilized for diagnostically corroborating details, including immunohistochemical findings. For comparison, histologic sections from 409 available $C Y L D$-wildtype $\mathrm{AC}$ were also evaluated.

Quantitative data were analyzed using the Fisher exact test owing to the categorical quality of the data and the size of the cohort. For comparing age and TMB between two groups, the nonparametric Mann Whitney $\mathrm{U}$ test was used. A two-tailed $P$ value of $<0.05$ was considered statistically significant, and the Bonferroni correction was applied for multiple simultaneous comparisons (Table 1).

\section{Review of publicly available dataset for corroboration in an independent cohort}

The AACR Project GENIE Consortium dataset (v7.0-public) [23] was interrogated for $\mathrm{AC}$ with mutations in $C Y L D$.

\section{Results}

\section{Clinical-pathologic features}

Of 574 consecutive nonadenocarcinoma AC specimens, 75 distinct cases (13\%) featured CYLD mutations. Compared with the rest of the AC cohort, the $C Y L D$-mutant group tended to be slightly younger (median 59 vs. 61 years, $p=$ 0.047 ) and was significantly enriched for female gender ( $88 \%$ vs. $67 \%, p=0.0001$ ) (Table 1 ). Nearly all cases were clinically advanced: most cases were stage IV $(n=63 / 75$; $84 \%$ ), while the remaining documented cases were stage IIA-B $(n=5)$ and IIIA-B $(n=3)$. Stage was unknown for four cases.

HPV status and typing were determined on all 574 patient samples. $C Y L D$-mutant cases showed near-universal detection of high-risk HPV sequences ( 73 out of 75 cases; 97\%), predominantly HPV16 (70/73; 96\%). A single HPV16 case had concurrent HPV33 reads, and the remaining three high-risk HPV cases contained HPV18 $(n=2)$ and HPV58 $(n=1)$ reads. $C Y L D$-mutant cases more frequently contained high-risk HPV reads compared with
CYLD-wildtype cases (97\% vs. $88 \%$ [440/499], $p=0.014$ ) (Table 1). CYLD-wildtype HPV-positive AC cases $(n=$ $440)$ also predominantly contained HPV16 reads $(n=388 /$ 440; $88 \%$ ).

Samples from the 75 CYLD-mutant cases consisted of 19 primary AC, two primary-site recurrences, and 54 metastatic disease samples. Of the metastatic samples, the majority were from liver $(n=29 / 54,54 \%)$, followed by lung $(n=15)$, distant lymph nodes $(n=3)$, a regional lymph node $(n=1)$, spleen $(n=1)$, bone $(n=1)$, duodenum $(n=1)$, omentum $(n=1)$, peritoneum $(n=1)$, and sacral soft tissue $(n=1)$. Compared with the $C Y L D$-wildtype $\mathrm{AC}$ cases in our cohort, $C Y L D$-mutant cases were sequenced much more frequently from metastases to the liver (39\% [29/75] vs. $18 \%$ [91/499], $p=0.0002)$ and lung (20\% [15/75] vs. $7 \%$ [35/499], $p=0.0008)$. Of the 75 CYLD-mutant AC samples, 56 consisted of core biopsies or small incisional biopsies, while 19 were excisional specimens.

Histopathologic examination of the CYLD-mutant carcinomas revealed a distinctive and predominant cylindromalike pattern (55/75 cases; $73 \%)$. Other cases showed basaloid morphology with squamous eddies $(5 / 75 ; 7 \%)$ and basaloid morphology without cylindroma-like features or discrete foci of squamous differentiation $(9 / 75 ; 12 \%)$. The remaining cases lacked basaloid cytomorphology and consisted of five conventional SCC with prominent keratinization and one carcinoma with transitional cell carcinomalike features.

The predominant pattern, cylindroma-like histomorphology, comprised a large majority of $C Y L D$-mutant $\mathrm{AC}$ (Fig. 1a-h). Histologic slides from 54 cases showed this pattern, while the pathology report from the single case without available slides for our review described matching features. Retrospective histologic examination of the 54 available cases revealed basaloid cells arranged in closely apposed, round to irregular tumor lobules that contained small, round, glassy, and eosinophilic inclusions of surrounding thickened basement membrane, reminiscent of cylindroma. While the basement membrane inclusions were present in all tumors in this subgroup, the inclusions were abundant in 44 cases $(81 \%$ ) (Fig. 1c, h) and focal in the other 10 cases (19\%) (Fig. 1f, g). The majority of the CYLD-mutant cylindroma-like AC cases showed necrosis (37 cases, 69\%), which was usually present in the center of tumor lobules in a comedonecrosis pattern (Fig. 1a, b, e). Only 10 of the $C Y L D$-mutant cylindroma-like cases (19\%) showed prominent peripheral palisading (Fig. 1d). Cytomorphology was basaloid in all $C Y L D$-mutant cylindromalike cases. Cytology was generally monomorphic, with only five cases (9\%) showing biphasic cytology with an admixture of basaloid cells and cells with slightly more cytoplasm and paler nuclei (Fig. 1h). Cellular 
Table 1 Comparative demographics, genomic alterations, and additional biomarkers of anal carcinomas stratified by $C Y L D$ mutation status, with $p$ values.

\begin{tabular}{|c|c|c|c|}
\hline & $C Y L D$-mutant & $C Y L D$-wildtype & $P$ \\
\hline Number of cases & 75 & 499 & \\
\hline$\%$ female & $88 \%$ & $67 \%$ & 0.0001 \\
\hline Median age (range) & $61(41-82)$ & $59(25-87)$ & 0.047 \\
\hline Cylindroma-like histology (\%) & $73 \%(55 / 75)$ & $8 \%(34 / 409)$ & $<0.0001$ \\
\hline Sequenced from liver metastasis $(\%)$ & $39 \%(29 / 75)$ & $18 \%(91 / 499)$ & 0.0002 \\
\hline Sequenced from lung metastasis $(\%)$ & $20 \%(15 / 75)$ & $7 \%(35 / 499)$ & 0.0008 \\
\hline Median TMB (Q1-Q3; mut/Mb) & $2.6(1.7-5.2)$ & $5.2(2.6-7.8)$ & $<0.00001$ \\
\hline High-risk HPV positive & $97 \%$ & $88 \%$ & 0.014 \\
\hline MSI high & $0 \%$ & $1 \%$ & 1.000 \\
\hline \multicolumn{4}{|l|}{ PI3K/AKT/mTOR pathway } \\
\hline$P I K 3 C A$ & $13 \%$ & $31 \%$ & 0.0015 \\
\hline$K M T 2 D^{\mathrm{a}}$ & $12 \%$ & $19 \%$ & 0.150 \\
\hline PTEN & $11 \%$ & $16 \%$ & 0.302 \\
\hline$F B X W 7$ & $5 \%$ & $14 \%$ & 0.041 \\
\hline STK11 & $1 \%$ & $5 \%$ & 0.232 \\
\hline$S O X 2^{\mathrm{a}}$ & $1 \%$ & $10 \%$ & 0.008 \\
\hline \multicolumn{4}{|l|}{ Epigenetic regulation } \\
\hline$K M T 2 C$ & $7 \%$ & $15 \%$ & 0.050 \\
\hline EP300 & $1 \%$ & $6 \%$ & 0.159 \\
\hline KDM6A & $4 \%$ & $5 \%$ & 1.000 \\
\hline$B A P 1$ & $1 \%$ & $3 \%$ & 0.712 \\
\hline \multicolumn{4}{|l|}{ DNA damage } \\
\hline TP53 & $9 \%$ & $12 \%$ & 0.568 \\
\hline \multicolumn{4}{|l|}{ Single pass transmembrane receptor } \\
\hline FAT1 & $3 \%$ & $9 \%$ & 0.048 \\
\hline NOTCH1 & $5 \%$ & $6 \%$ & 1.000 \\
\hline \multicolumn{4}{|l|}{ Cell cycle regulation } \\
\hline CCND1 (11q13.3) & $1 \%$ & $7 \%$ & 0.072 \\
\hline$C D K N 2 A$ & $1 \%$ & $6 \%$ & 0.106 \\
\hline$T E R T$ promoter & $1 \%$ & $5 \%$ & 0.157 \\
\hline$R B 1$ & $7 \%$ & $4 \%$ & 0.355 \\
\hline \multicolumn{4}{|l|}{ Wnt signaling } \\
\hline$C T N N B 1$ & $4 \%$ & $3 \%$ & 0.452 \\
\hline$A P C$ & $4 \%$ & $2 \%$ & 0.410 \\
\hline \multicolumn{4}{|l|}{ Receptor tyrosine kinase } \\
\hline$F G F R 3$ & $3 \%$ & $4 \%$ & 0.755 \\
\hline$E G F R$ & $1 \%$ & $3 \%$ & 0.706 \\
\hline \multicolumn{4}{|l|}{ RAS/MAPK pathway } \\
\hline$K R A S$ & $4 \%$ & $3 \%$ & 0.477 \\
\hline$B R A F$ & $3 \%$ & $<1 \%$ & 0.085 \\
\hline
\end{tabular}

The Bonferroni correction for 25 multiple simultaneous comparisons was applied; rows with a significant corrected $p$ value threshold $(<0.05 / 25=0.002)$ are in bold.

a Limited data in literature on role in PI3K/AKT signaling. pleomorphism was assessed to be mild in 40 cases (74\%), moderate in 12 cases (22\%), and severe in two cases (4\%). Rare squamous eddies were seen in three cases; otherwise, none demonstrated the presence of squamous eddies or pearls.
Because the cylindroma-like histomorphology within the $C Y L D$-mutant group was striking and potentially distinctive, we reviewed the histopathology from all 409 available $C Y L D$-wildtype $\mathrm{AC}$ in our database for comparison. Of the CYLD-wildtype cases, 407 were SCC, 


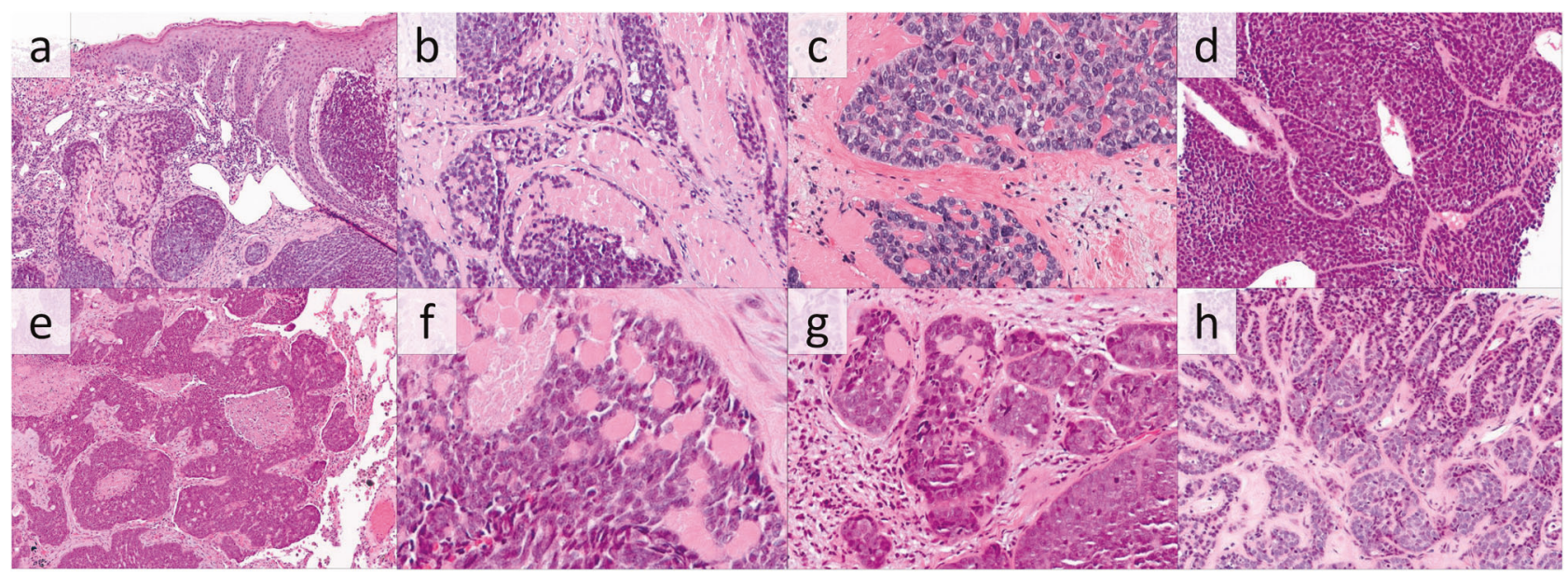

Fig. 1 Anal carcinomas with $C Y L D$ mutation and cylindroma-like histopathologic features. a Histopathologic examination reveals a carcinoma involving the dermis composed of rounded lobules of basaloid cells with hyaline inclusions and focally thickened basement membrane $(\mathrm{H} \& \mathrm{E}, 100 \times)$. b Higher power examination of this tumor reveals foci of necrosis and hyaline globules (H\&E, 200x). c Carcinoma with hyaline globules and associated thickened basement membrane (H\&E 400x) d Carcinoma with prominent jigsaw-like arrangement of basaloid cells with peripheral palisading, surrounding

including histologic variants, and two were adenocarcinomas. Only $8 \%(n=34)$ of the $C Y L D$-wildtype cases showed aggregates of tumor cells containing round fragments of hyalinized material, consistent with basement membrane inclusions, imparting a cylindroma-like appearance similar to the CYLD-mutant cases (Supplemental Fig. 1). These inclusions were abundant in 11 cases (32\%) and focal in 23 cases (68\%). The cytomorphology of CYLD-wildtype, cylindroma-like cases were predominantly basaloid (29 cases, $85 \%$ ), although five cases (15\%) consisted largely of squamoid cells with more voluminous, densely eosinophilic cytoplasm (Supplemental Fig. 1d). Importantly, compared with $C Y L D$-wildtype AC, $C Y L D$-mutant AC showed over ninefold higher frequency of cylindroma-like histology, as principally designated by the presence of basement membrane inclusions within tumor cell aggregates (73\% vs. $8 \%$, $p<0.0001$; Table 1).

Five $C Y L D$-mutant AC cases (7\%) were composed of aggregates of basaloid cells with squamous eddies (Fig. 2a, b). Squamous eddies, defined as rounded whorls of squamous cells with eosinophilic cytoplasm, were extensive in one case, focal in three cases, and rare in one case. A jigsaw-like arrangement of tumor lobules was present in two cases, while peripheral palisading was prominent in two. Necrosis was extensive in two cases and focal in one. No cases contained basement membrane inclusions or prominent basement membranes. Histologic features of squamous differentiation were limited to squamous eddies: squamous pearls were not identified, and intercellular spines were inconspicuous. Cellular pleomorphism was mild in all cases. thickened basement membrane, and small hyaline globules $(\mathrm{H} \& \mathrm{E}$, 200x). e, f This metastatic carcinoma involving the lung is composed of irregular lobules of basaloid cells with central necrosis and focal basement membrane inclusions (H\&E, 100×, and 400×). g Carcinoma with pleomorphic basaloid cells with focal hyaline globules (H\&E, 200x) (h) Carcinoma with thickened basement membrane and hyaline globules, also showing biphasic admixture of basaloid cells and cells with paler nuclei (H\&E, 200x).

Nine CYLD-mutant cases (12\%) consisted of basaloid cells with neither basement membrane inclusions nor squamous differentiation (Fig. 2c, d). Of these, six showed a jigsaw-like arrangement of tumor lobules, two showed prominent peripheral palisading, and four showed necrosis. Cellular pleomorphism was mild in three cases and moderate in six.

The remaining six cases of $C Y L D$-mutant $A C$ all lacked basaloid cytomorphology. Five cases (7\%) showed histologic features of conventional squamous cell carcinoma (Fig. 2e), with nodular proliferations of cells with dense, pink cytoplasm and severe nuclear pleomorphism. The remaining case showed transitional cell carcinoma-like histology, a recognized histologic pattern in AC [7], with thick trabeculae of tumor cells and abundant necrosis (Fig. 2f).

Glandular differentiation was not seen in any of the CYLD-mutant AC cases. The mucinous microcytic pattern, previously reported in AC [7] was not observed, and clear cell changes were not present in any of the CYLDmutant cases.

Although immunohistochemical slides were not available for confirmatory review by the authors, accompanying pathology reports stated that the CYLD-mutant ACs that were evaluated by immunohistochemistry were nearly always positive for p63 (30/31 cases), p40 (15/16 cases), CK5/6 (26/27 cases), and p16 (24/24 cases). In contrast, CK7 was diffusely positive in only $6 / 20$ cases, focally positive in $4 / 20$ cases, and negative in the remaining 10 cases. $C Y L D$-mutant ACs were uniformly negative when stained for synaptophysin 


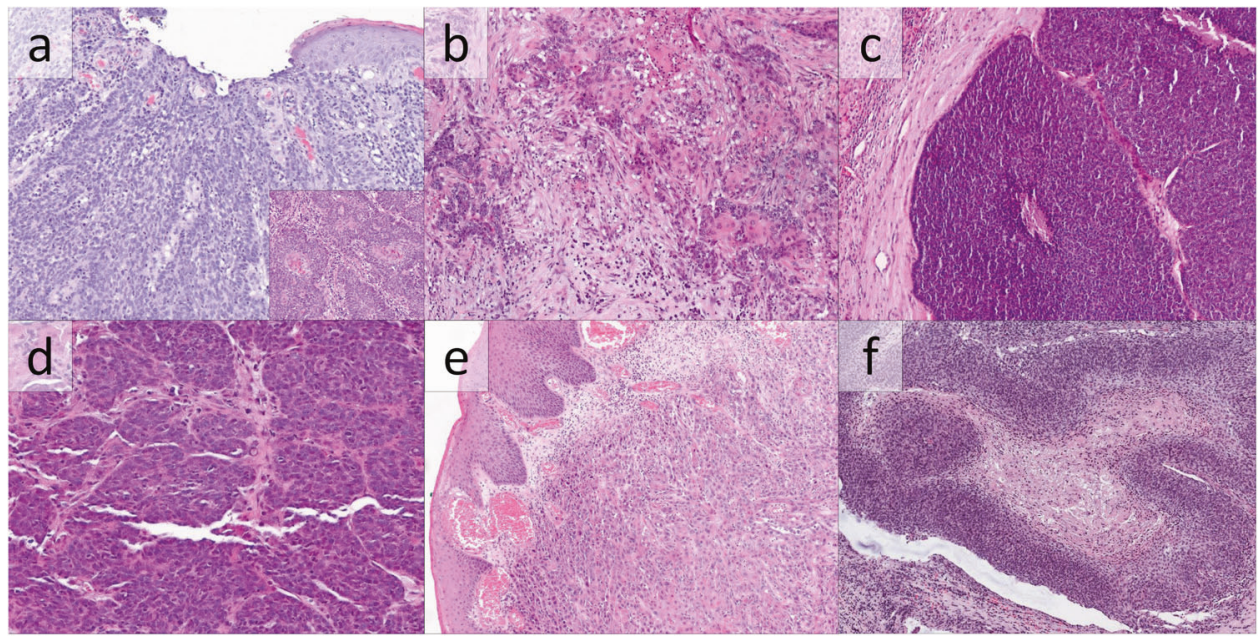

Fig. 2 Anal carcinomas with $C Y L D$ mutation lacking cylindromalike basement membrane inclusions. (a and inset) Anal carcinoma composed predominantly of irregular aggregates of basaloid cells showing rare continuity with surface epithelium (a) and focal squamous eddies (inset) (H\&E, 200×). b Metastatic anal basaloid carcinoma involving a lymph node with infiltrative small aggregates of basaloid cells with occasional squamous morules (H\&E 200x). c Basaloid anal carcinoma, metastatic to liver, consisting of large aggregates of basaloid tumor cells without apparent basement

( $n=24$ cases), chromogranin $(n=20)$, CK20 $(n=20)$, CDX2 $(n=13)$, and TTF1 $(n=13)$.

\section{Comprehensive genomic profiling}

$C Y L D$ mutations included truncating $(n=50)$, homozygous deletion $(n=10)$, missense $(n=16)$, and splice site $(n=3)$ mutations (Fig. 3a, b). Truncating mutations occurred in exons $4(n=2), 5(n=5), 8(n=1), 9(n=10), 10(n=5)$, $11(n=3), 12(n=3), 13(n=1), 15(n=3), 16(n=4), 17$ $(n=3), 18(n=3), 19(n=2)$, and $20(n=5)$. Missense mutations occurred in exons $4(n=3), 8(n=1), 10(n=2)$, $12(n=4), 15(n=1), 17(n=2), 18(n=1), 19(n=1)$, and $20(n=1)$.

Figure $3 \mathrm{a}$ and Table 1 show the most frequent genomic alterations outside the $C Y L D$ locus in the $C Y L D$-mutant vs. $C Y L D$-wildtype AC cohort. The CYLD-mutant group showed significantly lower TMB and less frequent alterations in PIK3CA (Table 1).

Cases with cylindroma-like histomorphology $(n=55)$ showed significantly lower TMB compared with cases with basaloid histology lacking cylindroma-like features $(n=14)$ (1.7 vs. $4.4, p=0.0058)$. In addition, cases with basaloid histology lacking cylindroma-like features showed a higher frequency of PTEN alterations (29\% [4/14] vs. 5\% [3/55], $p=0.0273$ ). Demographics of cylindroma-like and noncylindroma-like basaloid cases were similar.

Further analysis revealed that, compared with cases with basaloid histomorphology without further differentiation membrane material or squamous differentiation (H\&E, 100x). d Basaloid anal carcinoma with small, closely apposed aggregates of basaloid tumor cells without apparent basement membrane inclusions or squamous differentiation (H\&E, 200x). e Anal carcinoma composed of aggregates of cells with glassy eosinophilic cytoplasm, consistent with conventional squamous cell carcinoma (H\&E, 100×). f A single case showed thickened trabeculae of basaloid carcinoma cells with a transitional cell carcinoma-like appearance (H\&E, 100x).

$(n=9)$, cases with basaloid appearance with focal squamous differentiation $(n=5)$ had a significantly higher TMB (7.8 vs. $3.5, p=0.0105$ ). No other significant differences were identified.

Compared with the remainder of the CYLD-mutant cohort, cases with conventional squamous cell carcinoma histology with prominent keratinization ( $n=5$ cases) were associated with HPV-negative status (40\% [2/5] vs. $0 \%$ [0/ 70 ], $p=0.0036)$ and showed elevated TMB (median $=5.2$ vs. 2.6, $p=0.0242$ ). Cases also showed a significantly elevated frequency of alterations in $F B X W 7$ (40\% [2/5] vs. $2 / 70$ [3\%], $p=0.0204)$, FATl $(40 \%$ [2/5] vs. $0 \%$ [0/70], $p=0.0036)$, and KMT2C (40\% [2/5] vs. 4\% [3/70], $p=0.0331$ ).

Tumors sequenced from primary $(n=21)$ vs. metastatic sites $(n=54)$ showed similar demographics and percentages of genomic alterations. Median TMB, while slightly lower for tumors sequenced from primary sites, was not significantly different (1.7 vs. $2.6 \mathrm{mut} / \mathrm{Mb}, p=0.428$ ).

Mutational signatures were obtained for nine $C Y L D$ mutant cases. Three cases were identified with an APOBEC signature (two with basaloid histology, one with TCC-like histology). Available $C Y L D$-wildtype cases $(n=162)$ showed similar frequency of APOBEC signature $(n=91 /$ 162).

Comparison of cases with $C Y L D$ mutations within the ubiquitin-specific protease domain (exons 12-20) and outside the ubiquitin hydrolase domain (exons 4-11) revealed no significant differences. 
a

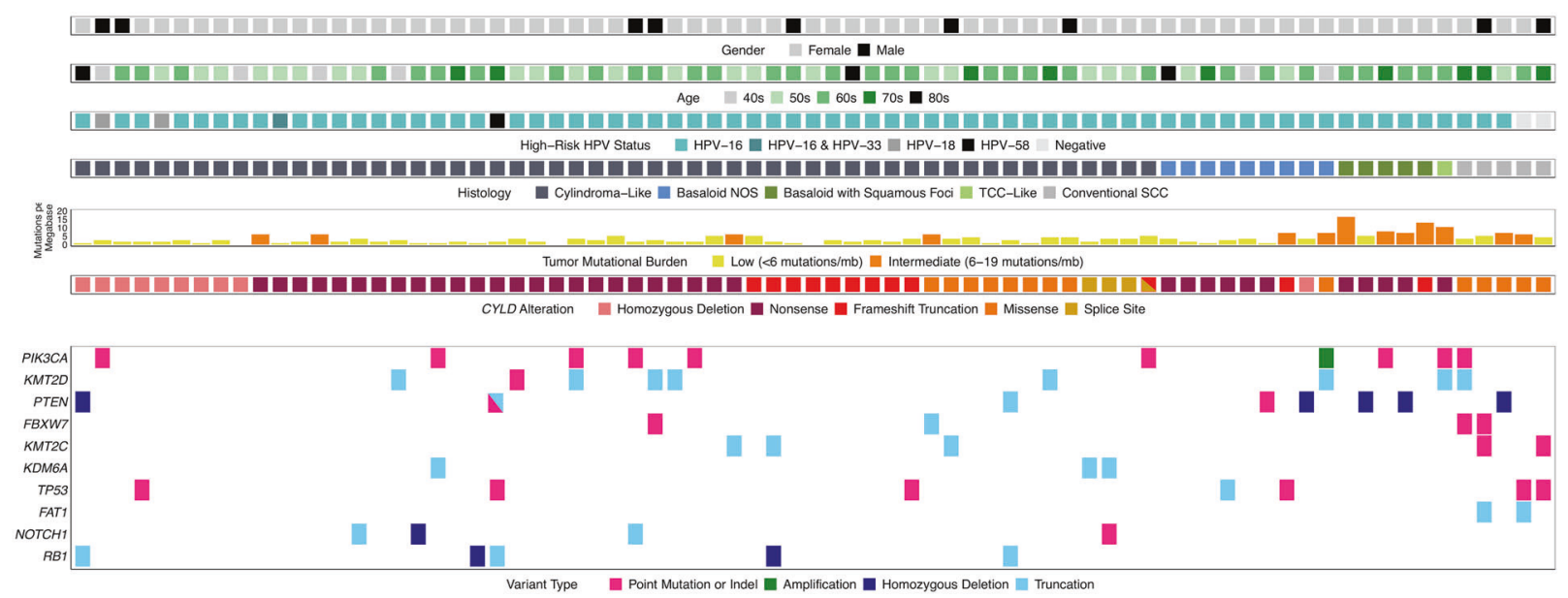

b

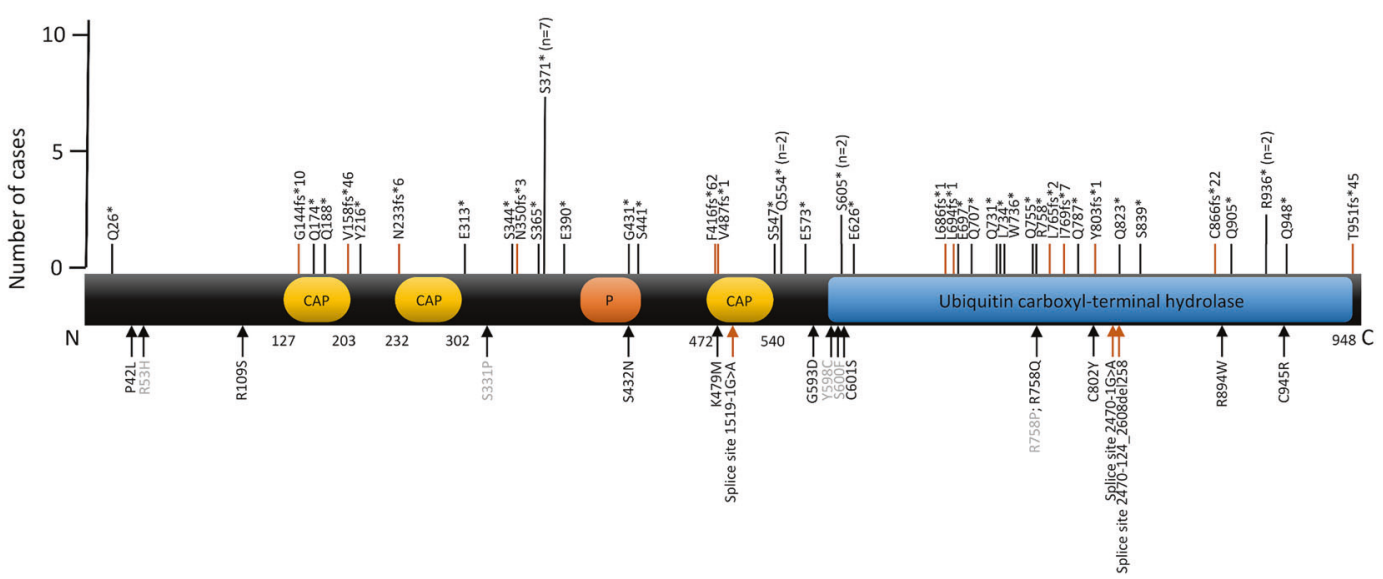

Fig. 3 Clinicopathologic features and molecular landscape of CYLD-mutant anal carcinoma. a Tile plot to summarize clinical features, histopathology, and molecular alterations in $C Y L D$-mutant anal carcinoma. b Schematic of functional domains of CYLD (transcript NM_015247), to include identified mutation sites (79 mutations in 75 cases). The resulting terminal amino acid location for nonsense $(n=38)$ and frameshift mutations $(n=12)$ in $C Y L D$ are denoted by

In total, 17 of the 75 cases had available SGZ algorithm data to predict germline status of $C Y L D$ alterations. Of the 17 cases, two cases had two $C Y L D$ mutations each. In total, 18 of the 19 CYLD mutations were predicted to be somatic. One case with a single $C Y L D$ mutation $(\mathrm{P} 42 \mathrm{~L}$; allele frequency $=60 \%$ ) was predicted germline; the case showed cylindroma-like histology.

Comparison of $C Y L D$-wildtype AC with vs. without cylindroma-like histology revealed that cylindroma-like cases showed significantly lower frequency of PIK3CA (9\% [3/34] v. 32\% [120/375], $p=0.0032)$ and PTEN genomic alterations $(3 \%[1 / 34]$ vs. $18 \%$ [66/375], $p=$ 0.027 ), and higher frequency of sequencing from samples of liver metastasis $(32 \%$ [11/34] vs. $17 \%$ [63/375], $p=0.034)$. 32 of 34 CYLD-wildtype cylindroma-like AC (94\%) were black and orange bars, respectively (top of diagram). Missense mutations $(n=16)$ and splice site mutations $(n=3)$, each identified in single case, are labeled with black and orange arrows, respectively (lower diagram). Mutations in gray font were identified in cases with conventional squamous cell carcinoma histology. Ten cases with homozygous deletions in CYLD are not shown in (b). CAP cytoskeleton-associated proteins, $\mathrm{P}$ phosphorylation region.

positive for high-risk HPV. Thus, in these key parameters, $C Y L D$-wildtype cylindroma-like AC resembled $C Y L D$ mutant cylindroma-like AC. No other significant differences between $C Y L D$-wildtype AC with vs. without cylindromalike histology were identified.

\section{Publicly available dataset for corroboration in an independent cohort}

The unexpectedly high frequency of $C Y L D$-mutant cases in our AC cohort (13\%) prompted us to interrogate the AACR Project GENIE Consortium dataset (v7.0-public) [23]. A total of 10 CYLD-mutant AC cases were identified (10/83 [12.0\%]), nearly identical to our $13 \%$. Median age was 58 years (range 51-80), and the cases were enriched for female 
gender $(n=8 / 10)$, as in our cohort. CYLD mutations included truncating $(n=6)$, homozygous deletion $(n=2)$, and missense $(n=2)$ mutations. Truncating mutations occurred in exons $9(n=1), 11(n=1), 12(n=1), 17(n=2)$, and 18 $(n=1)$. Missense mutations occurred in exons $5(n=1)$ and $20(n=1)$. As in our cohort, concurrent alterations were each of low frequency: in PTEN ( $n=1$ case), KMT2D $(n=1$ case), BRAF ( $n=1$ case), APC ( $n=1$ case), and RBI $(n=1$ case). All cases were wildtype for $P I K 3 C A$ alterations. Histopathology was not available for review.

\section{Discussion}

Our series connects a distinctive histopathology of cylindroma-like basaloid carcinoma of the anus with mutations in $C Y L D$, a gene responsible for both syndromic [8] and sporadic [9] cylindromas, confirming both a morphologic and genetic relationship with cutaneous cylindroma. Furthermore, the close correlation of HPV-positivity and $C Y L D$ mutation in this context supports the concept that $C Y L D$ mutation contributes to AC primarily through HPVrelated carcinogenesis.

Previous studies have reported rare anal basaloid carcinomas with "adenoid cystic carcinoma-like" histologic features, including dense globules of basement membrane material within tumor lobules [5-7]. Among these histopathologic studies, Chetty et al. [6] described two cases that closely resemble the cases in our series. Those authors noted both the "jigsaw" pattern of intimately admixed tumor lobules and the presence of basement membrane material highly reminiscent of cylindroma. Photomicrographs from their article [6] and a later article by Graham et al. [7] show prominent eosinophilic, glassy thickening of the basement membrane surrounding tumor lobules with small round inclusions of the same material within tumor lobules, closely resembling cylindroma-like carcinomas in this study. Interestingly, a detailed case report of spiradenocylindromalike basaloid carcinoma of the anus showed highly similar histologic features to our cases and those of references $[6,7]$, as well as HPV positivity, but examination of the CYLD gene detected no mutation in the tumor [24]; we similarly identified cylindroma-like carcinomas infrequently among CYLD-wildtype ACs. Another recent report described a similar case, designated as cylindroma-like basaloid AC, although molecular studies were not described [25]. Immunohistochemistry results extracted from pathology reports in our cohort were similar to the cases in previous studies [6, 7], with CK5/6, p63, and p16 positivity, and variable $\mathrm{CK} 7$ positivity.

Although several investigators have noted the histologic resemblance of reportedly rare $\mathrm{ACs}$ to cylindroma $[6,24,25]$, to our knowledge, $C Y L D$ mutation has not been described in detail for AC. Comprehensive molecular genetic studies of AC have shown various mutations in HPV-positive cases, especially those involving the phosphoinositol-3-kinase pathway (including $P I K 3 C A$, $P T E N$, and $A K T 1 / 2$ ), while HPV-negative carcinomas often show TP53 mutation and loss of CDKN2A [26-31]. A search of AC in the AACR Project GENIE Consortium dataset [23] found that $12 \%$ of ACs harbor CYLD mutations, similar to the $13 \%$ incidence in our cohort.

CYLD functions as a deubiquitinating enzyme which negatively regulates activity of the $\mathrm{NF}-\kappa \mathrm{B}$ pathway $[11,12]$ and c-Jun N-terminal kinase pathway [13-15]. In mouse models, inactivation of CYLD in the epidermis promotes sebaceous hyperplasia and proliferations with basaloid and sebaceous components [32], while global knockout mice are prone to developing cutaneous papillomas [12]. Loss of CYLD activity has been correlated with more aggressive behavior in cutaneous squamous cell carcinoma [33], melanoma [13, 34], pancreatic carcinoma [35], and hepatocellular carcinoma [15]. In addition, gene expression profiling of CYLD-mutant tumors has shown dysregulated tropomyosin kinase signaling, which has been suggested as a potential target for therapy [36, 37].

The preponderance of clinical studies of CYLD mutation have concerned syndromic manifestations of familial cylindromatosis, Brooke-Spiegler syndrome, and multiple familial trichoepitheliomas. After the initial characterization of CYLD mutation in familial cylindromatosis [8], subsequent publications identified a variety of mutations in these cutaneous tumor syndromes $[38,39]$. A recent review of the literature described 95 unique syndrome-associated mutations of CYLD, most occurring within exons 9-20 [40] and most resulting in protein truncation [40]. CYLD mutations are also present in sporadic cylindromas and some spiradenomas [9]. Most CYLD mutations in our AC cohort are similar, i.e., occurring within exons 9-20 and resulting in protein truncation. In addition, germline prediction results suggest that the $C Y L D$ mutations in $\mathrm{AC}$ were predominantly somatic.

A recent molecular genetic study of cutaneous cylindromas, spiradenomas, and spiradenocarcinomas found CYLD mutations in nearly all cylindromas and some spiradenomas [9]. Most spiradenomas, however, harbored ALPK1 mutations, which were mutually exclusive from CYLD mutations. Spiradenocarcinomas showed frequent oncogenic comutations involving genes such as TP53. That study did not investigate cylindrocarcinoma, an extremely rare tumor.

Previously reported cylindrocarcinomas of the skin are not clearly analogous to the cases described in our current series. Cutaneous cylindrocarcinomas of the skin show heterogeneous malignant components, ranging from low grade adenocarcinoma resembling that arising from the 
salivary gland to sarcomatoid malignant tumors, only identifiable as related to the adnexal tumor by the presence of an intact precursor cylindroma by histologic examination [41]. Prior studies of the genetics of cylindrocarcinoma are limited; investigations have to-date described TP53 mutations in small numbers of cylindrocarcinomas within larger series of adnexal carcinomas [42, 43].

While CYLD-mutated ACs share some histologic features with cutaneous cylindroma, multiple histologic differences are readily apparent. Both tend to show a jigsawlike arrangement of basaloid cell aggregates with thickened basement membranes and inclusions of basement membrane material. CYLD-mutant basaloid carcinomas, however, show permeative growth and frequently display necrosis, often in a comedo pattern, features unusual for cutaneous cylindroma. In addition, while cutaneous cylindromas are typically composed of a dimorphic cell population, with dark cells containing scant cytoplasm at the periphery of tumor lobules and light cells with pale cytoplasm and nuclei centrally, the CYLD-mutated basaloid carcinomas in our series were typically composed of monomorphic populations of basaloid cells. Finally, the degree of nuclear irregularity, hyperchromasia, and pleomorphism seen in the $C Y L D$-mutant ACs exceeds that of cutaneous cylindroma.

Recent molecular genetic study of HPV-positive head and neck SCC found that one third of cases harbored mutation of either CYLD or TRAF3, the latter of which also encodes a deubiquitinating enzyme and negative regulator of NF- $\mathrm{KB}$ [10]. Among HPV-positive head and neck SCC, CYLD- or TRAF3-mutant tumors showed increased NF-kB activity, episomal HPV DNA rather than integrated HPV DNA, increased expression of genes related to adhesion, motility, proliferation, and differentiation, and improved survival. $C Y L D$ and TRAF3 mutations were found only rarely among HPV-negative head and neck SCC, suggesting that CYLD mutation contributes to carcinogenesis mostly through HPVrelated pathways. While these studies lacked histomorphologic data which would enable closer correlation with the cases in our cohort, the demonstrated relationship between CYLD mutation and HPV-driven carcinogenesis may be analogous to our series of CYLD-mutant cylindroma-like ACs.

In this study, the presence of cylindroma-like histologic appearance, as principally defined by the presence of basement membrane inclusions, was significantly correlated with CYLD mutation in the context of AC. Cylindroma-like histology had a sensitivity and specificity of $73 \%$ and $92 \%$, respectively, for the presence of CYLD mutation. Notably, among cylindroma-like cases, hyaline globule inclusions tended to be abundant in the $C Y L D$-mutant group and focal in the $C Y L D$-wild type group. There were also some cases of $C Y L D$-wild type AC with cylindroma-like inclusions, but with more squamoid cytology (Supplemental Fig. 1d), differing from the $C Y L D$-mutant cases. The $C Y L D$-mutant cases without accompanying cylindroma-like features may be partly attributable to sampling error; most biopsies were partial and may have missed cylindroma-like foci. With respect to the $C Y L D$-wildtype cases with cylindroma-like hyaline globule inclusions, we have considered that these cases may contain non-CYLD, as-yet uncharacterized mutations activating related pathways to promote a similar histogenesis, although this requires additional study.

The rarity of HPV-negative ACs with CYLD mutation suggests that HPV infection is an obligate precursor for CYLD-mutant basaloid carcinomas of the anus. Intriguingly, in contrast to cutaneous sites, where cylindromas are far more common than their malignant counterpart, a benign counterpart to cylindroma-like basaloid carcinoma of the anus is not known. To our knowledge, cylindromas, which occur most commonly on the scalp, are extremely rare in the anal or perianal region [44].

CYLD mutations also characterize a subset of salivary gland neoplasms: both basal cell salivary adenomas and adenocarcinomas can possess $C Y L D$ mutations [45]. Interestingly, the basal cell adenomas and carcinomas harbor mutations of $C Y L D$ in exons 9-11, while only basal cell adenocarcinomas harbor $C Y L D$ mutations involving exons 12-20, corresponding to the ubiquitin-specific hydrolase domain. Comparison of ACs with CYLD mutations in exons 9-11 and exons 12-20 revealed no significant differences. Of note, the rare reported cases of basal cell adenocarcinoma with HPV testing were HPV(-) and, therefore, perhaps not analogous to $C Y L D$-mutant AC [46].

Other recent work has refined the understanding of a particular subtype of sinonasal carcinoma, which shows both adenoid cystic carcinoma-like histologic features and HPV-positivity [47, 48]. This carcinoma, designated "HPVpositive multiphenotypic sinonasal carcinoma", pairs histologic features of a spectrum of salivary gland carcinomas with involvement of surface epithelium [48]. Interestingly, in addition to displaying cribriform patterns highly reminiscent of adenoid cystic carcinoma, some cases also contain foci of hyalinized basement membrane-like material within tumor cell aggregates. CYLD mutation status of these lesions has not been described to the authors' knowledge.

The distinctive nature of our underlying sample set is a limitation of this study. All cases were sent for CGP for detection of targetable genetic alterations. As such, the cases tended towards advanced disease in which surgical therapy alone was not curative. Although we lacked detailed followup to definitively characterize the full clinical significance of $C Y L D$ mutation, we documented a significant preponderance of liver involvement amongst $C Y L D$-mutant AC.

The majority of our cases were core biopsies, and, thus, limited sampling may have precluded identification of some histologic features (e.g., additional material from a case 
with the basaloid pattern without further differentiation may have revealed cylindroma-like features). Although some primary tumor samples contained portions of intact mucosa enabling corroboration of anal site, the anatomical designation of primary site was based principally on accompanying requisition forms and pathology reports. While we reviewed data on immunohistochemistry from accompanying pathology reports, we were not able to review immunohistochemical slides to confirm reported findings or to perform additional immunostains. In addition, while comparative sequencing of normal tissue for definitive exclusion of germline mutation was not available, the SGZ algorithm findings were consistent with somatic mutations in $94 \%$ of cases with available data.

CYLD-mutant cylindroma-like basaloid carcinomas of the anus represent a significant subgroup of ACs with distinctive genetic and histologic features and near-universal detection of HPV-positivity. These characteristic genetic findings, paired with the striking histopathology, may aid in the subtyping of $\mathrm{AC}$ [1]. Our findings represent a genotypephenotype correlation with potentially important implications for classification of AC. Additional studies are needed to define prognostic features and potential therapeutic approaches $[36,37]$ to this unique, common, and previously uncharacterized, anal cancer subtype.

Acknowledgements We would like to acknowledge the American Association for Cancer Research and its financial and material support in the development of the AACR Project GENIE registry, as well as members of the consortium for their commitment to data sharing. Interpretations are the responsibility of study authors.

\section{Compliance with ethical standards}

Conflict of interest EAW, MM, RS, DCP, ESS, BA, JV, JAE, JSR, SHR, ACH, and JYT are employees of Foundation Medicine, Inc., a wholly owned subsidiary of Roche Holdings, Inc. and Roche Finance Ltd, and these employees have equity interest in an affiliate of these Roche entities. KJW receives research funding from Novo Nordisk for studies unrelated to cancer. JC, PJP, BJG, and MCM declare no conflict of interest.

Publisher's note Springer Nature remains neutral with regard to jurisdictional claims in published maps and institutional affiliations.

Open Access This article is licensed under a Creative Commons Attribution 4.0 International License, which permits use, sharing, adaptation, distribution and reproduction in any medium or format, as long as you give appropriate credit to the original author(s) and the source, provide a link to the Creative Commons license, and indicate if changes were made. The images or other third party material in this article are included in the article's Creative Commons license, unless indicated otherwise in a credit line to the material. If material is not included in the article's Creative Commons license and your intended use is not permitted by statutory regulation or exceeds the permitted use, you will need to obtain permission directly from the copyright holder. To view a copy of this license, visit http://creativecommons. org/licenses/by/4.0/.

\section{References}

1. Graham R. Anal squamous cell carcinoma. In: Goldblum J, Klimstra D, Lam A, editors. Digestive system tumours WHO classification of tumours. 5th ed. Lyon, France: International Agency for Research on Cancer; 2019. p. 205-7.

2. Yang EJ. Human papilloma virus-associated squamous neoplasia of the lower anogenital tract. Surg Pathol Clin. 2019;12:263-79.

3. Ju JY, Stelow EB. Clinicopathologic features of anal and perianal squamous cell carcinomas and their relationship to human papillomavirus. Am J Surg Pathol. 2019;43:827-34.

4. Wong AK, Chan RC, Aggarwal N, Singh MK, Nichols WS, Bose $\mathrm{S}$. Human papillomavirus genotypes in anal intraepithelial neoplasia and anal carcinoma as detected in tissue biopsies. Mod Pathol. 2010;23:144-50.

5. Klotz RG, Pamukcoclu T, Souilliard DH. Transitional cloacogenic carcinoma of the anal canal. Cancer. 1966;20:1727-45.

6. Chetty R, Serra S, Hsieh E. Basaloid squamous carcinoma of the anal canal with an adenoid cystic pattern: histologic and immunohistochemical reappraisal of an unusual variant. Am J Surg Pathol. 2005;29:1668-72.

7. Graham RP, Arnold CA, Naini BV, Lam-Himlin DM. Basaloid squamous cell carcinoma of the anus revisited. Am J Surg Pathol. 2016;40:354-60.

8. Bignell GR, Warren W, Seal S, Takahashi M, Rapley E, Barfoot $\mathrm{R}$, et al. Identification of the familial cylindromatosis tumoursuppressor gene. Nat Genet. 2000;25:160-5.

9. Rashid M, van der Horst M, Mentzel T, Butera F, Ferreira I, Pance A, et al. ALPK1 hotspot mutation as a driver of human spiradenoma and spiradenocarcinoma. Nat Commun. 2019;10:1-10.

10. Hajek M, Sewell A, Kaech S, Burtness B, Yarbrough WG, Issaeva N. TRAF3/CYLD mutations identify a distinct subset of human papillomavirus-associated head and neck squamous cell carcinoma. Cancer. 2017;123:1778-90.

11. Ikeda F, Dikic I. CYLD in ubiquitin signaling and tumor pathogenesis. Cell. 2006;125:643-5.

12. Massoumi R, Chmielarska K, Hennecke K, Pfeifer A, Fässler R. Cyld Inhibits tumor cell proliferation by blocking Bcl-3dependent NF-кB signaling. Cell. 2006;125:665-77.

13. Ke H, Augustine CK, Gandham VD, Jin JY, Tyler DS, Akiyama $\mathrm{SK}$, et al. CYLD inhibits melanoma growth and progression through suppression of the jnk/ap-1 and $\beta 1$-integrin signaling pathways. J Invest Dermatol. 2013;133:221-9.

14. Sato Y, Goto E, Shibata Y, Kubota Y, Yamagata A, Goto-Ito S, et al. Structures of CYLD USP with Met1-or Lys63-linked diubiquitin reveal mechanisms for dual specificity. Nat Struct Mol Biol. 2015;22:222-9.

15. Nikolaou K, Tsagaratou A, Eftychi C, Kollias G, Mosialos G, Talianidis I. Inactivation of the deubiquitinase CYLD in hepatocytes causes apoptosis, inflammation, fibrosis, and cancer. Cancer Cell. 2012;21:738-50.

16. Frampton GM, Fichtenholtz A, Otto GA, Wang K, Downing SR, $\mathrm{He} \mathbf{J}$, et al. Development and validation of a clinical cancer genomic profiling test based on massively parallel DNA sequencing. Nat Biotechnol. 2013;31:1023-31.

17. Sun JX, He Y, Sanford E, Montesion M, Frampton GM, Vignot S, et al. A computational approach to distinguish somatic vs. germline origin of genomic alterations from deep sequencing of cancer specimens without a matched normal. PLoS Pathog. 2018;14:e1005965.

18. Chalmers ZR, Connelly CF, Fabrizio D, Gay L, Ali SM, Ennis R, et al. Analysis of 100,000 human cancer genomes reveals the landscape of tumor mutational burden. Genome Med. 2017;9:34.

19. Trabucco SE, Gowen K, Maund SL, Sanford E, Fabrizio DA, Hall $\mathrm{MJ}$, et al. A novel next-generation sequencing approach to 
detecting microsatellite instability and pan-tumor characterization of 1000 microsatellite instability-high cases in 67,000 patient samples. J Mol Diagn. 2019;21:1053-66.

20. Muñoz N, Bosch FX, De Sanjosé S, Herrero R, Castellsagué X, Shah KV, et al. Epidemiologic classification of human papillomavirus types associated with cervical cancer. N Engl J Med. 2003;348:518-27.

21. Zehir A, Benayed R, Shah RH, Syed A, Middha S, Kim HR, et al. Mutational landscape of metastatic cancer revealed from prospective clinical sequencing of 10,000 patients. Nat Med. 2017;23:703-13

22. Amin MB, Edge S, Greene F, Byrd DR, Brookland RK, Washington MK, et al. AJCC cancer staging system. 8th ed. New York: Spinger; 2017.

23. Sweeney SM, Cerami E, Baras A, Pugh TJ, Schultz N, Stricker T, et al. AACR project genie: powering precision medicine through an international consortium. Cancer Disco. 2017;7:818-31.

24. Kacerovska D, Szepe P, Vanecek T, Nemcova J, Michal M, Mukensnabl P, et al. Spiradenocylindroma-like basaloid carcinoma of the anus and rectum: case report, including HPV studies and analysis of the CYLD gene mutations. Am J Dermatopathol. 2008;30:472-6.

25. Chen S, Tan SA, Shon W, Shaw CM. Cylindroma-like basaloid anal cancer presenting as a large pelvic mass in a patient with ulcerative colitis. J Surg Case Rep. 2015;2015:rjv093.

26. Bernardi MP, Ngan SY, Michael M, Lynch AC, Heriot AG, Ramsay RG, et al. Molecular biology of anal squamous cell carcinoma: Implications for future research and clinical intervention. Lancet Oncol. 2015;16:e611-21.

27. Chung JH, Sanford E, Johnson A, Klempner SJ, Schrock AB, Palma NA, et al. Comprehensive genomic profiling of anal squamous cell carcinoma reveals distinct genomically defined classes. Ann Oncol. 2016;27:1336-41.

28. Morris V, Rao X, Pickering C, Foo WC, Rashid A, Eterovic K, et al. Comprehensive genomic profiling of metastatic squamous cell carcinoma of the anal canal. Mol Cancer Res. 2017; 15:1542-50.

29. Cacheux W, Dangles-Marie V, Rouleau E, Lazartigues J, Girard E, Briaux A, et al. Exome sequencing reveals aberrant signalling pathways as hallmark of treatment-naive anal squamous cell carcinoma. Oncotarget. 2018;9:464-76.

30. Cacheux W, Rouleau E, Briaux A, Tsantoulis P, Mariani P, Richard-Molard $\mathrm{M}$, et al. Mutational analysis of anal cancers demonstrates frequent PIK3CA mutations associated with poor outcome after salvage abdominoperineal resection. Br J Cancer. 2016;114:1387-94.

31. Schrock AB, Pavlick D, Klempner SJ, Chung JH, Forcier B, Welsh A, et al. Hybrid capture-based genomic profiling of circulating tumor DNA from patients with advanced cancers of the gastrointestinal tract or anus. Clin Cancer Res. 2018;24:1881-90.

32. Jin YJ, Wang S, Cho J, Selim MA, Wright T, Mosialos G, et al. Epidermal CYLD inactivation sensitizes mice to the development of sebaceous and basaloid skin tumors. JCI Insight. 2016;1:1-11.

33. Alameda JP, Moreno-Maldonado R, Navarro M, Bravo A, Ramírez A, Page A, et al. An inactivating CYLD mutation promotes skin tumor progression by conferring enhanced proliferative, survival and angiogenic properties to epidermal cancer cells. Oncogene. 2010;29:6522-32.
34. de Jel MM, Schott M, Lamm S, Neuhuber W, Kuphal S, Bosserhoff AK. Loss of CYLD accelerates melanoma development and progression in the $\mathrm{Tg}(\mathrm{Grm} 1)$ melanoma mouse model. Oncogenesis. 2019;8:56.

35. Xie S, Wu Y, Hao H, Li J, Guo S, Xie W, et al. CYLD deficiency promotes pancreatic cancer development by causing mitotic defects. J Cell Physiol. 2019;234:9723-32.

36. Rajan N, Elliott R, Clewes O, MacKay A, Reis-Filho JS, Burn J, et al. Dysregulated TRK signalling is a therapeutic target in CYLD defective tumours. Oncogene. 2011;30:4243-60.

37. Danilenko M, Stamp E, Stocken DD, Husain A, Zangarini M, Cranston A, et al. Targeting tropomyosin receptor kinase in cutaneous CYLD defective tumors with pegcantratinib the TRAC randomized clinical trial.: JAMA Dermatol. 2018; 154:913-21.

38. Almeida S, Maillard C, Itin P, Hohl D, Huber M. Five new CYLD mutations in skin appendage tumors and evidence that aspartic acid 681 in CYLD is essential for deubiquitinase activity. J Invest Dermatol. 2008;128:587-93.

39. Nasti S, Pastorino L, Bruno W, Gargiulo S, Battistuzzi L, Zavattaro E, et al. Five novel germline function-impairing mutations of CYLD in Italian patients with multiple cylindromas. Clin Genet. 2009;76:481-5.

40. Nagy N, Farkas K, Kemény L, Széll M. Phenotype-genotype correlations for clinical variants caused by CYLD mutations. Eur J Med Genet. 2015;58:271-8.

41. Kazakov DV, Zelger B, Rütten A, Vazmitel M, Spagnolo DV, Kacerovska D, et al. Morphologic diversity of malignant neoplasms arising in preexisting spiradenoma, cylindroma, and spiradenocylindroma based on the study of 24 cases, sporadic or occurring in the setting of brooke-spiegler syndrome. Am J Surg Pathol. 2009;33:705-19.

42. Kazakov DV, Grossmann P, Spagnolo DV, Vanecek T, Vazmitel M, Kacerovska D, et al. Expression of p53 and TP53 mutational analysis in malignant neoplasms arising in preexisting spiradenoma, cylindroma, and spiradenocylindroma, sporadic or associated with brooke-spiegler syndrome. Am J Dermatopathol. 2010;32:215-21.

43. Biernat W, Peraud A, Wozniak L, Ohgaki H. P53 mutations in sweat gland carcinomas. Int J Cancer. 1998;76:317-20.

44. Baker GM, Selim MA, Hoang MP. Vulvar adnexal lesions: a 32year, single-institution review from Massachusetts General Hospital. Arch Pathol Lab Med. 2013;137:1237-46.

45. Rito M, Mitani Y, Bell D, Mariano FV, Almalki ST, Pytynia KB, et al. Frequent and differential mutations of the CYLD gene in basal cell salivary neoplasms: linkage to tumor development and progression. Mod Pathol. 2018;31:1064-72.

46. Haeggblom L, Ursu RG, Mirzaie L, Attoff T, Gahm C, Nordenvall $\mathrm{LH}$, et al. No evidence for human papillomavirus having a causal role in salivary gland tumors. Diagn Pathol. 2018;13:44.

47. Bishop JA, Ogawa T, Stelow EB, Moskaluk CA, Koch WM, Pai SI, et al. Human papillomavirus-related carcinoma with adenoid cystic-like features: a peculiar variant of head and neck cancer restricted to the sinonasal tract. Am J Surg Pathol. 2013;37:836-44.

48. Bishop JA, Andreasen S, Hang JF, Bullock MJ, Chen TY, Franchi A, et al. HPV-related multiphenotypic sinonasal carcinoma. Am J Surg Pathol. 2017;41:1690-701. 


\section{Affiliations}

Erik A. Williams ${ }^{1}{ }^{1} \cdot$ Meagan Montesion ${ }^{1}{ }^{1} \cdot$ Radwa Sharaf $^{1} \cdot$ James Corines $^{2} \cdot$ Parth J. Patel $^{3} \cdot$ Brendan J. Gillespie $^{1} \cdot$ Dean C. Pavlick ${ }^{1} \cdot$ Ethan S. Sokol ${ }^{1} \cdot$ Brian M. Alexander $^{1} \cdot K_{\text {Kevin Jon Williams }}^{4} \cdot$ Julia A. Elvin $^{1} \cdot$ Jeffrey S. Ross $^{1,2}$. Shakti H. Ramkissoon ${ }^{1,5} \cdot$ Amanda C. Hemmerich $^{1} \cdot$ Julie Y. Tse $^{1,6} \cdot$ Mark C. Mochel (D) $^{7}$

1 Foundation Medicine, Inc., 150 Second Street, Cambridge, MA 02141, USA

2 Department of Pathology, State University of New York Upstate Medical University, 766 Irving Avenue, Syracuse, NY 13210, USA

3 Department of Surgery, Lewis Katz School of Medicine at Temple University, Philadelphia, PA 19140, USA

4 Department of Physiology and Medicine, Lewis Katz School of Medicine at Temple University, Philadelphia, PA 19140, USA
5 Wake Forest Comprehensive Cancer Center and Department of Pathology, Wake Forest School of Medicine, Winston-Salem, NC 27157, USA

6 Department of Pathology \& Laboratory Medicine, Tufts University School of Medicine, 145 Harrison Ave, Boston, MA 02111, USA

7 Departments of Pathology and Dermatology, Virginia Commonwealth University School of Medicine, Richmond, VA 23298, USA 